\title{
Comprehensive analysis of differentially expressed profiles of non-coding RNAs in peripheral blood and ceRNA regulatory networks in non-syndromic orofacial clefts
}

\author{
YUWEI GAO ${ }^{1}$, QIGUANG ZANG ${ }^{1}$, HONGQUAN SONG ${ }^{1}$, SONGBIN FU ${ }^{2}$, WENJING SUN ${ }^{2}$, \\ WEI ZHANG ${ }^{3}$, XIAOTONG WANG ${ }^{1}$, YONG LI $^{1}$ and XIAOHUI JIAO ${ }^{1}$ \\ ${ }^{1}$ Department of Oral Maxillofacial Surgery, The First Affiliated Hospital, Harbin Medical University, \\ Harbin, Heilongjiang 150001; ${ }^{2}$ Laboratory of Medical Genetics, Harbin Medical University, Harbin, \\ Heilongjiang 150081; ${ }^{3}$ Department of Oral Maxillofacial Surgery, The Fourth Affiliated Hospital, \\ Harbin Medical University, Harbin, Heilongjiang 150001, P.R. China
}

Received November 4, 2018; Accepted April 11, 2019

DOI: $10.3892 / \mathrm{mmr} .2019 .10261$

\begin{abstract}
Non-syndromic orofacial clefts (NSOC), which include cleft lip with or without cleft palate $(\mathrm{CL} / \mathrm{P})$ and cleft palate only (CPO), are common congenital birth defects in humans. Accumulating evidence indicates that long non-coding RNAs (lncRNAs) and microRNAs (miRNAs or miRs) play important roles in NSOC; however, the potential regulatory associations between them remain largely unknown. In this study, we performed next-generation RNA sequencing (RNA-seq) to identify transcriptome profiles, including mRNAs, lncRNAs and miRNAs, in patients with CL/P and CPO. A total of 36 lncRNAs, 1,341 mRNAs and 60 miRNAs were found to be differentially expressed in the CL/P group compared to the control group, and 57 lncRNAs, 1,255 mRNAs and 162 miRNAs were found to be differentially expressed in the CPO group compared to the control group. Subsequently, reverse transcription-quantitative
\end{abstract}

Correspondence to: Professor Xiaohui Jiao, Department of Oral Maxillofacial Surgery, The First Affiliated Hospital, Harbin Medical University, 23 Youzheng Street, Nangang, Harbin, Heilongjiang 150001, P.R. China

E-mail: jiaoxiaohui_dds@163.com

Abbreviations: NSOC, non-syndromic orofacial clefts; CL/P, cleft lip with or without cleft palate; $\mathrm{CPO}$, cleft palate only; lncRNA, long non-coding RNA; miRNA, microRNA; ncRNA, non-coding RNA; ceRNA, competing endogenous RNA; RNA-seq, next-generation RNA sequencing; GWAS, genome-wide association study; RT-qPCR, reverse transcription-quantitative polymerase chain reaction; GO, gene ontology; KEGG, Kyoto Encyclopedia of Genes and Genomes; BP, biological process; CC, cellular component; $\mathrm{MF}$, molecular function; MRE, miRNA response elements; FPKM, fragments per kilobase million; DE, differentially expressed

Key words: long non-coding RNA, microRNA, mRNA, RNA sequencing, ceRNA, non-syndromic orofacial clefts polymerase chain reaction (RT-qPCR) was performed to validate the expression of selected IncRNAs, miRNAs and mRNAs. In addition, bioinformatics methods were employed to explore the potential functions of ncRNAs and to construct IncRNA-miRNA-mRNA regulatory networks. To the best of our knowledge, this is the first study to comprehensively analyze regulated non-coding RNAs (ncRNAs) in CL/P and $\mathrm{CPO}$, providing a novel perspective on the etiology of NSOC and laying the foundation for future research into the potential regulatory mechanisms of ncRNAs and mRNAs in NSOC.

\section{Introduction}

Non-syndromic orofacial clefts (NSOC) are common congenital birth defects in humans, with a worldwide incidence of approximately 1 in 700 cases. The etiology of NSOC is complex, and is generally believed to involve interactions between environmental and genetic factors. NSOC can be divided into cleft lip with or without cleft palate (CL/P) and cleft palate only (CPO) according to different etiologies $(1,2)$.

Previous research has focused on the etiological mechanisms underlying NSOC, although much remains to be discovered (3-5). A number of genome-wide association studies (GWAS) have revealed that several gene variants can lead to NSOC; however, these protein-coding genes can only partially explain the causes of NSOC (6-8). The Human Genome Project found that only $2 \%$ of genes in the genome code for proteins. Moreover, previous GWAS data analyses have found that approximately $80 \%$ of susceptible sites are not in protein coding regions (9), illustrating that genes that do not code for proteins also play important roles in the incidence of NSOC.

Non-coding RNA (ncRNA) is a general term for a variety of RNA types that do not encode proteins; nevertheless, these RNAs regulate gene expression on multiple levels. ncRNAs can be grouped according to their length. MicroRNAs (miRNAs or miRs) are a kind of endogenous small RNAs with a length of approximately 19-25 nt that can regulate the expression of genes at the transcriptional level (10). miRNAs can degrade or 
inhibit the translation of target genes by binding specifically to their 3'-UTRs. Mounting evidence indicates that ncRNAs play important roles in various diseases, such as cancer, cardiovascular diseases and nervous system diseases, as well as others (11-13). In recent years, several researchers have found that ncRNAs also affect the pathogenesis of NSOC. For example, during lip development, the target genes of the miR-203 and miR-302 clusters are different subtypes of p63, and it has been shown that the deletion of these genes can cause different degrees of CL/P (14). In addition, a functional analysis of zebrafish models found that the overexpression of miR-23b led to the broadening of the ethmoid plate and aberrant cartilage structures in the viscerocranium, while the overexpression of miR-133b causes a reduction in ethmoid plate size and a significant midfacial cleft (15).

Long non-coding RNAs (lncRNAs) are ncRNAs with a length of $>200 \mathrm{nt}$ with no protein coding function. They regulate the expression of genes at the transcriptional, post-transcriptional and epigenetic levels (16). lncRNAs have drawn increasing public attention in recent years; increasing evidence suggests that they play crucial regulatory roles in various physiological and pathological processes. However, reports of the functions and mechanisms of action of lncRNAs as regards the development of the lip and palate are limited. The lncRNA H19 gene was initially identified as a CPO-related gene in transforming growth factor (TGF) $\beta 3$-knockout mice by RNA sequencing analysis (17). Based on this finding, the present study investigated the expression of lncRNA H19 in mice with a cleft palate induced by 2,3,7,8-tetrachlorodibenzo-p-dioxin (TCDD) and retinoic acid (RA). The results revealed that the expression of lncRNA H19 and its target gene, insulin like growth factor 2 (IGF2), changed from E13.5 to E15.5, exhibiting a negative correlation. This finding indicated that lncRNA H19 may interact with its target gene, IGF2, playing significant roles in palate development $(18,19)$. Although lncRNAs and miRNAs can regulate gene expression, their roles are not independent, and they form a complex regulatory network.

Although emerging data have revealed that ncRNAs and mRNAs play important roles in NSOC, no studies of the potential interactions between ncRNAs and mRNAs in the development of NSOC have been reported to date, at least to the best of our knowledge. Using high-throughput sequencing methods, this study identified differentially expressed (DE) ncRNAs and mRNAs from the peripheral blood of patients with $\mathrm{CL} / \mathrm{P}$ and $\mathrm{CPO}$. Using bioinformatics analyses, we then predicted the function and potential regulatory associations of the ncRNAs, with a goal of discovering novel etiological molecular mechanisms of NSOC.

\section{Materials and methods}

Sample collection. This study was approved by the Ethics Committee of Harbin Medical University (Harbin, China), and informed consent was obtained from the patients' families before sampling. The participants were divided into 3 groups as follows: A CL/P group, a CPO group and a healthy control group. The selection criteria were the following: Patients with a cleft lip and palate, without other congenital malformations and genetic diseases, who were hospitalized at the First
Affiliated Hospital of Harbin Medical University were selected for this study. All 36 participants included in this study were aged between 1 month and 40 months and enrolled from January 2017 to May 2018. On the morning of the 2nd day of admission, $5 \mathrm{ml}$ of peripheral blood were collected using tubes containing EDTA. In this study, 9 samples (3 controls, 3 CL/P and $3 \mathrm{CPO}$ ) were used for RNA-Seq analysis, and another 27 samples (10 controls, $10 \mathrm{CL} / \mathrm{P}$ and $7 \mathrm{CPO}$ ) were used for reverse transcription-quantitative polymerase chain reaction (RT-qPCR). Participant information is presented in Table I.

RNA extraction and quality control. Total RNA was extracted from the samples using TRIzol ${ }^{\circledR}$ reagent (Thermo Fisher Scientific, Inc., Waltham, MA, USA) according to the manufacturer's instructions. RNA degradation and contamination were monitored using $1 \%$ agarose gels. RNA purity was examined using a NanoPhotometer ${ }^{\circledR}$ spectrophotometer (Implen, Inc., Westlake Village, CA, USA). The RNA concentration was measured using the Qubit ${ }^{\circledR}$ RNA Assay kit in a Qubit ${ }^{\circledR} 2.0$ Fluorometer (Thermo Fisher Scientific, Inc.). RNA integrity was assessed using the RNA Nano 6000 Assay kit for the Bioanalyzer 2100 system (Agilent Technologies, Santa Clara, CA, USA).

Construction of the RNA sequencing library. A total of $3 \mu \mathrm{g}$ of RNA per sample was used as input material for the RNA sample preparations. First, ribosomal RNA was removed using an Epicentre Ribo-zero ${ }^{\mathrm{TM}}$ rRNA Removal kit (Epicentre, Charlotte, NC, USA) and rRNA-free residue was cleaned by ethanol precipitation. Subsequently, sequencing libraries were generated from the rRNA-depleted RNA using a NEBNext ${ }^{\circledR}$ Ultra $^{\mathrm{TM}}$ Directional RNA Library Prep kit for Illumina ${ }^{\circledR}$ (NEB, Ipswich, MA, USA), following the manufacturer's recommendations. Briefly, fragmentation was carried out using divalent cations under elevated temperature in NEBNext First Strand Synthesis Reaction Buffer (5X). First-strand cDNA was synthesized using a random hexamer primer and M-MuLV Reverse Transcriptase (RNase H-). Second strand cDNA synthesis was subsequently performed using DNA Polymerase I and RNase H. In the reaction buffer, dNTPs with dTTP were replaced by dUTP. Remaining overhangs were converted into blunt ends via exonuclease/polymerase activity. Following the adenylation of the 3' ends of DNA fragments, NEBNext Adaptors with hairpin loop structures were ligated to prepare for hybridization. To select cDNA fragments that were preferentially $150-200 \mathrm{bp}$ in length, the library fragments were purified with the AMPure XP System (Beckman Coulter, Beverly, MA, USA). Subsequently, $3 \mu 1$ of USER Enzyme (NEB) was used with the size-selected, adaptor-ligated cDNA at $37^{\circ} \mathrm{C}$ for $15 \mathrm{~min}$ followed by $5 \mathrm{~min}$ at $95^{\circ} \mathrm{C}$ before PCR. PCR was performed with Phusion High-Fidelity DNA polymerase, universal PCR primers and the Index (X) Primer. Finally, products were purified (AMPure XP System), and library quality was assessed on the Agilent Bioanalyzer 2100 system. (Agilent Technologies).

Construction and sequencing of small RNA libraries. A total of $3 \mu \mathrm{g}$ of total RNA per sample was used as the input material for the small RNA libraries. Sequencing libraries were generated using the NEBNext ${ }^{\circledR}$ Multiplex Small RNA Library Prep 
Table I. Information regarding the participants.

\begin{tabular}{|c|c|c|c|}
\hline Group & Age, months & Sex & Sample use \\
\hline $\mathrm{CL} / \mathrm{P}$ & 3 & Male & RNA-seq \\
\hline $\mathrm{CL} / \mathrm{P}$ & 10 & Male & RNA-seq \\
\hline $\mathrm{CL} / \mathrm{P}$ & 11 & Female & RNA-seq \\
\hline $\mathrm{CL} / \mathrm{P}$ & 12 & Male & RT-qPCR \\
\hline $\mathrm{CL} / \mathrm{P}$ & 9 & Female & RT-qPCR \\
\hline $\mathrm{CL} / \mathrm{P}$ & 5 & Male & RT-qPCR \\
\hline $\mathrm{CL} / \mathrm{P}$ & 12 & Male & RT-qPCR \\
\hline $\mathrm{CL} / \mathrm{P}$ & 27 & Male & RT-qPCR \\
\hline $\mathrm{CL} / \mathrm{P}$ & 18 & Male & RT-qPCR \\
\hline $\mathrm{CL} / \mathrm{P}$ & 10 & Female & RT-qPCR \\
\hline $\mathrm{CL} / \mathrm{P}$ & 8 & Male & RT-qPCR \\
\hline $\mathrm{CL} / \mathrm{P}$ & 6 & Male & RT-qPCR \\
\hline $\mathrm{CL} / \mathrm{P}$ & 4 & Male & RT-qPCR \\
\hline $\mathrm{CPO}$ & 24 & Male & RNA-seq \\
\hline $\mathrm{CPO}$ & 18 & Female & RNA-seq \\
\hline $\mathrm{CPO}$ & 14 & Male & RNA-seq \\
\hline $\mathrm{CPO}$ & 15 & Female & RT-qPCR \\
\hline $\mathrm{CPO}$ & 8 & Female & RT-qPCR \\
\hline $\mathrm{CPO}$ & 11 & Male & RT-qPCR \\
\hline $\mathrm{CPO}$ & 35 & Female & RT-qPCR \\
\hline $\mathrm{CPO}$ & 37 & Female & RT-qPCR \\
\hline $\mathrm{CPO}$ & 16 & Female & RT-qPCR \\
\hline $\mathrm{CPO}$ & 15 & Female & RT-qPCR \\
\hline Control & 9 & Male & RNA-seq \\
\hline Control & 7 & Male & RNA-seq \\
\hline Control & 5 & Male & RNA-seq \\
\hline Control & 6 & Male & RT-qPCR \\
\hline Control & 4 & Male & RT-qPCR \\
\hline Control & 1 & Female & RT-qPCR \\
\hline Control & 4 & Female & RT-qPCR \\
\hline Control & 5 & Female & RT-qPCR \\
\hline Control & 11 & Female & RT-qPCR \\
\hline Control & 4 & Female & RT-qPCR \\
\hline Control & 23 & Female & RT-qPCR \\
\hline Control & 5 & Female & RT-qPCR \\
\hline Control & 5 & Female & RT-qPCR \\
\hline
\end{tabular}

RT-qPCR, reverse transcription-quantitative polymerase chain reaction; RNA-seq, next-generation RNA sequencing; CL/P, cleft lip with or without cleft palate; $\mathrm{CPO}$, cleft palate only.

Set for Illumina ${ }^{\circledR}$ (NEB) following the manufacturer's recommendations and index codes were added to attribute sequences for each sample. Briefly, NEB 3' SR Adaptors were directly and specifically ligated to the $3^{\prime}$ end of the miRNA. Following the 3 ' ligation reaction, the SR RT Primer hybridized to the excess 3' SR Adaptor (that remained free after the 3' ligation reaction) and transformed the single-stranded DNA adaptor into a double-stranded DNA molecule. This step is important to prevent adaptor-dimer formation. Furthermore, dsDNAs are not substrates for ligation mediated by T4 RNA Ligase 1 and therefore would not ligate to the 5' SR Adaptor in the subsequent ligation step. The 5' ends adapter was ligated to the 5 ' ends of the miRNAs. First-strand cDNA was then synthesized using M-MuLV Reverse Transcriptase (RNase H-). PCR amplification was performed using LongAmp Taq 2X Master Mix, SR Primer for Illumina and the Index (X) primer (NEB). PCR products were purified on an $8 \%$ polyacrylamide gel $(100 \mathrm{~V}, 80 \mathrm{~min})$. DNA fragments corresponding to 140-160 bp (the length of the small ncRNA plus the $3^{\prime}$ and $5^{\prime}$ adaptors) were recovered and dissolved in $8 \mu \mathrm{l}$ of elution buffer. Finally, library quality was assessed on an Agilent Bioanalyzer 2100 system using DNA High Sensitivity Chips. Clustering of the index-coded samples was performed on a cBot Cluster Generation System using a TruSeq SR Cluster kit v3-cBot-HS (Illumina) according to the manufacturer's instructions. Following cluster generation, the library preparations were sequenced on an Illumina HiSeq 2500 platform (Illumina, Inc., San Diego, CA, USA), and 125-bp paired-end and 50-bp single-end reads were generated.

Analysis of the sequencing results. Low-quality raw reads containing adapters were filtered to get clean reads. For lncRNAs, the clean reads were aligned to the reference genome using HISAT2 (version 2.0.4) (20), and then the mapped reads of each sample were assembled by StringTie software (version 1.3.1) (20,21). A series of strict screening conditions was then set according to the structure and functional characteristics to identify lncRNAs for the following analysis. The screening conditions included: Number of exons $\geq 2$, length $>200$ bp, fragments per kilobase million (FPKM) $\geq 0.5$, and removing transcripts that overlap the exon region of the database annotation and with coding potential by either/all of the four tools: CNCI version 2 (22), CPC version cpc-0.9-r2 (23), Pfam-sca version 1.3 (24) and phyloCSF version 20121028 (25). FPKMs of both lncRNAs and coding genes in each sample were calculated for differential expression analysis using Ballgown software with a P-adjust $<0.05$ (20). For miRNAs, the clean reads were screened to include those with a length of 18-25 nt and were aligned to the reference sequence using Bowtie software (version bowtie-0.12.9) (26), which was combined with miREvo software (version miREvo_v1.1) (27) and miRDeep2 software (version mirdeep2_0_0_5) (28) to analyze the functions of new miRNAs and adopt DESeq2 (version 1.12.0) (29) with a negative binomial distribution to analyze DE miRNAs. All sequencing programs were carried out by Novogene Company (China, Beijing).

$R T-q P C R$ validation. To validate the reliability of the RNAseq results, 2 miRNAs (miR-483-3p and miR-92b-5p), 2 lncRNAs (lncRNA RP11-731F5.2 and lncRNA XIST) and 2 mRNAs [BCL2 associated athanogene 5 (BAG5) and zinc finger E-box binding homeobox 2 (ZEB2)] were selected for further validation using the RT-qPCR method with another 27 samples (10 controls, $10 \mathrm{CL} / \mathrm{P}$ and $7 \mathrm{CPO}$ ). The selection criteria were based on the fold changes and P-values of DE genes, and the selection was also based on the DE genes that have been associated with NSOC. The total RNA of each sample was extracted, and first-strand cDNA was synthesized using cDNA Synthesis kit (GK8030; Generay Biotech Co., Ltd., Shanghai, China) and RevertAid First Strand cDNA Synthesis kit (K1621 
and EN0521; Thermo Fisher Scientific, Inc.) according to the manufacturer's instructions. The temperature protocol used for reverse transcription reaction was as follows: Incubation for $5 \mathrm{~min}$ at $25^{\circ} \mathrm{C}$, followed by $60 \mathrm{~min}$ at $42^{\circ} \mathrm{C}$ and terminating the reaction by heating at $70^{\circ} \mathrm{C}$ for $5 \mathrm{~min}$. The cDNA was then used as the template for a RT-qPCR reaction with Power SYBR $^{\circledR}$ Green PCR Master Mix (cat. no. 4367659; Applied Biosystems, Thermo Fisher Scientific, Inc.) and Power qPCR PreMix (GK8020; Generay Biotech Co., Ltd.). Reactions were performed in 3 independent wells. The thermocycling conditions for qPCR were as follows: Initial denaturation at $95^{\circ} \mathrm{C}$ for $10 \mathrm{~min}$, followed by 40 cycles at $95^{\circ} \mathrm{C}$ for $10 \mathrm{sec}$ and annealing at $60^{\circ} \mathrm{C}$ for $34 \mathrm{sec}$. The sequences of the primers are presented in Table II. The relative expression levels of miRNAs, mRNAs and IncRNAs were calculated using miR-16-5p and $\beta$-actin as internal references. The RT-qPCR results were quantified using the $2^{-\Delta \Delta \mathrm{Cq}}$ method (30).

Analysis of the miRNA-mRNA-lncRNA regulatory network. lncRNAs have extensive regulatory functions. Not only can they directly regulate the structure of DNA and the transcription and translation of RNA, but they can also regulate gene expression indirectly by acting as miRNA sponges by competitively binding to the same miRNA binding sites with mRNAs. Based on the theory of competing endogenous RNA (ceRNA), we attempted to look for lncRNA-gene pairs with the same binding sites as miRNAs to construct lncRNA-miRNA-mRNA regulatory networks with lncRNA as a decoy, miRNA as a center and mRNA as a target, and we visualized these networks using Cytoscape software (http://www.cytoscape.org/download.php).

Prediction and functional analysis of lncRNA target genes. IncRNAs can regulate the expression of target genes by colocalization and co-expression. We set the threshold of colocalization as $100 \mathrm{~kb}$ upstream and downstream of the lncRNAs. Pearson correlation coefficients with absolute values $>0.95$ were used to concurrently analyze the correlation between IncRNAs and mRNAs. The target genes of DE miRNAs were predicted using the intersection of the miRanda (http://miranda.org.uk/), PITA (https://genie.weizmann. ac.il/pubs/mir07/mir07_prediction.html) and RNAhybrid databases (https://bibiserv.cebitec.uni-bielefeld.de/rnahybrid/). Subsequently, the candidate target genes obtained from the above methods were analyzed by Gene Ontology (GO, http://www.geneontology.org/) and Kyoto Encyclopedia of Genes and Genomes (KEGG, https://www.genome.jp/kegg/) pathway enrichment analysis using GOseq (version 2.12) (31) and KOBAS (version 2.0) (32), respectively. GO is an international standard classification system for gene function. According to the distribution of predicted genes, which are identified in each group in GO, the functions of DE ncRNAs were predicted and classified. The groups include biological process (BP), cellular component (CC) and molecular function (MF). Different genes coordinate with each other to perform their biological functions in vivo. Significant pathway enrichment can determine the main biochemical metabolic pathways and signal transduction pathways involving target genes. KEGG is the main public database of pathways and uses the KEGG pathway as a unit, applying hypergeometric
Table II. Primers designed for reverse transcription-quantitative polymerase chain reaction validation of candidate noncoding RNAs and mRNAs.

\begin{tabular}{ll}
\hline Gene & \multicolumn{1}{c}{ Primer sequence (5'-3') } \\
\hline ZEB2 & F: CAAGCACCACCTTATCGAGC \\
& R: TGTGATTCATGTGCTGCGAG \\
BAG5 & F: CACATCCTTCCGTTGCCAAA \\
& R: CCCGAGAGCACACAGGATAA \\
XIST & F: GACTACCCAAAGCCCCTTCT \\
& R: AGTCAACACTGCACCAACAC \\
RP11-731F5.2 & F: CAGTCAAGACCATCGCCAAG \\
& R: CCACTGGTCCCATCACTTCT \\
ß-actin & F: GTCCACCTTCCAGCAGATGT \\
& R: CTCAGTAACAGTCCGCCTAGAA \\
hsa-miR-483-3p & F: GCGCTCACTCCTCTCCTC \\
& R: TGGGTTCATTTCTGGGTCTT \\
hsa-miR-92b-5p & F: GCGCAGGGACGGGACGCGG \\
& R: TGGGTTCATTTCTGGGTCTT \\
hsa-miR-16-5p & F: GCGCTAGCAGCACGTAAAT \\
& R: TGGGTTCATTTCTGGGTCTT
\end{tabular}

ZEB2, zinc finger E-box binding homeobox 2; BAG5, BCL2 associated athanogene 5; F, forward; R, reverse.

tests to define significantly enriched pathways involving target genes in comparison to the whole genome background.

Statistical analysis. All measurement data are presented as the means \pm SD. The relative expression levels of miR-92b-5p and IncRNA XIST between the CPO and control groups were analyzed by Student's t-test using GraphPad Prism ${ }^{\circledR}$ version 7.04 software (GraphPad Software, Inc., La Jolla, CA, USA). The relative expression levels of miR-483-3p, lncRNA RP11-731F5.2, BAG5 and ZEB2 in the CPO, CL/P and control groups were analyzed by one-way ANOVA with Dunnett's multiple comparisons test using GraphPad Prism Version 7.04 software (GraphPad Software, Inc.). $\mathrm{P}<0.05$ was considered to indicate a statistically significant difference.

\section{Results}

DE ncRNAs and mRNAs. A total of 3,450 annotated lncRNAs, 1,447 annotated miRNAs, 3,990 novel lncRNAs and 97 novel miRNAs were obtained after mapping to the reference genome and carrying out prediction according to the structure and functional characteristics of lncRNAs and miRNAs. We analyzed ncRNAs and mRNAs according to fold change values and significance levels to identify DE lncRNAs, miRNAs and mRNAs. The distribution of DE miRNAs (Fig. 1A-C), DE lncRNAs (Fig. 2A-C) and DE mRNAs (Fig. 3A-C) was directly visualized using volcano plots. The results were as follows: There were $36 \mathrm{DE}$ lncRNAs (15 upregulated and 21 downregulated), 1,341 DE mRNAs (778 upregulated and 563 downregulated), and 60 


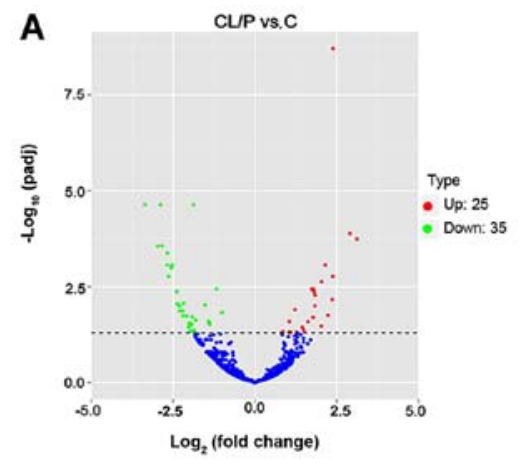

D
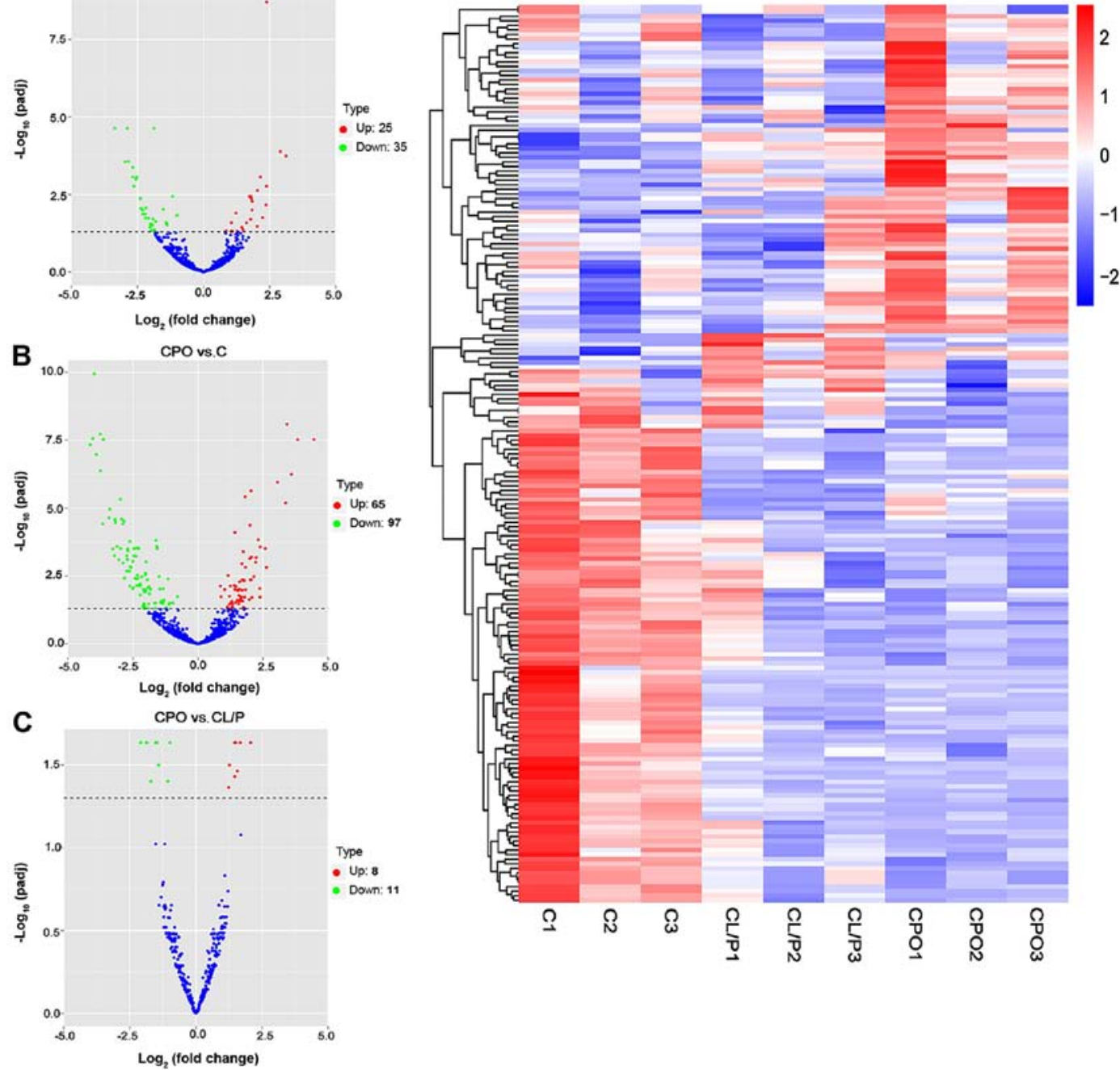

Figure 1. Transcriptome profile of RNA-Seq data of the miRNAs for the 3 groups. (A) Volcano plots of DE miRNAs between the CL/P and control groups, (B) between the $\mathrm{CPO}$ and control groups, and (C) between the CL/P and CPO groups. Red and green dots represent upregulated and downregulated miRNAs, respectively. (D) Hierarchical clustering of the expression profiles of DE miRNAs in the control, CL/P and CPO groups. miRNA, microRNA; CL/P, cleft lip with or without cleft palate; $\mathrm{CPO}$, cleft palate only; $\mathrm{C}$, the healthy control group.

DE miRNAs (25 upregulated and 35 downregulated) in the CL/P group compared to the control group. There were $57 \mathrm{DE}$ IncRNAs (22 upregulated and 35 downregulated), 1,255 DE mRNAs (634 upregulated and 621 downregulated), and 162 DE miRNAs (65 upregulated and 97 downregulated) in the CPO group compared to the control group. There were 18 DE IncRNAs (9 upregulated and 9 downregulated), 761 DE mRNAs (213 upregulated and 548 downregulated) and 19 DE miRNAs (8 upregulated and 11 downregulated) in the $\mathrm{CPO}$ group compared to the $\mathrm{CL} / \mathrm{P}$ group. Since there were a number of DE genes obtained from RNA-seq in the study, detailed information of the top 10 DE miRNAs, lncRNAs and mRNAs in the CL/P group compared to the control group and in the CPO group compared to the control group is presented in Tables III-VIII. Hierarchical clustering analysis was used to reveal the expression profiles of DE miRNAs (Fig. 1D), DE lncRNAs (Fig. 2D) and DE mRNAs (Fig. 3D) in the control, $\mathrm{CL} / \mathrm{P}$ and $\mathrm{CPO}$ groups.

$R T-q P C R$ validation of $n c R N A$ and $m R N A$ expression. To validate the reliability of the sequencing results and to provide evidence for further functional experiments with the DE ncRNAs we identified, the expression levels of 6 genes, including miR-483-3p, miR-92b-5p, BAG5, ZEB2, lncRNA RP11-731F5.2 and IncRNA XIST, were analyzed by RT-qPCR along with another 27 samples (10 CL/P, 7 CPO and 10 healthy controls). The expression levels of each gene according to RT-qPCR and RNAseq are presented in Fig. 4. With the exception of ZEB2, the expression trends of these genes according to RT-qPCR were almost the same as those obtained with RNAseq, indicating that the sequencing results were reliable.

Analysis of miRNA-lncRNA-mRNA regulatory networks. Based on the theory of ceRNA, miRNA-lncRNA-mRNA regulatory networks were constructed and visualized using Cytoscape software in the CL/P (Fig. 5) and CPO groups (Fig. 6). Thus, at the whole-transcriptome level, the possible regulatory mechanism between ncRNAs and mRNAs was revealed in the CL/P and CPO groups. As shown in the figures, crucial ncRNAs and mRNAs with more edges may play important roles in the pathology of $\mathrm{CPO}$ and $\mathrm{CL} / \mathrm{P}$; these ncRNAs and mRNAs include miR-483-3p, miR-4690-3p, 

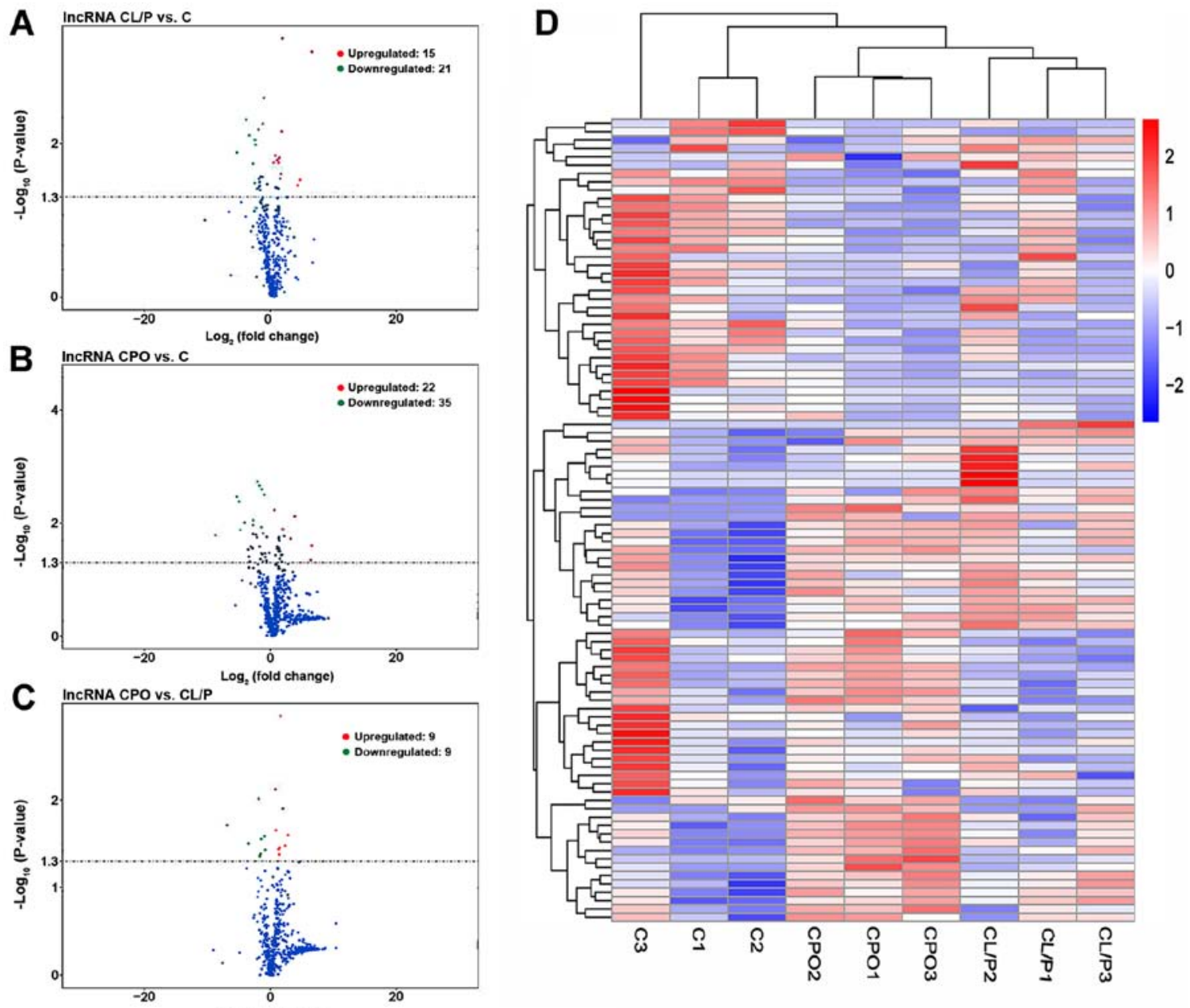

Figure 2. Transcriptome profile of RNA-Seq data of the lncRNAs for the 3 groups. (A) Volcano plots of DE lncRNAs between the CL/P and control groups, (B) between the $\mathrm{CPO}$ and control groups, and (C) between the $\mathrm{CL} / \mathrm{P}$ and CPO groups. Red and green dots represent upregulated and downregulated lncRNAs, respectively. (D) Hierarchical clustering of the expression profiles of DE lncRNAs in the control, CL/P and CPO groups. IncRNA, long non-coding RNA; $\mathrm{CL} / \mathrm{P}$, cleft lip with or without cleft palate; $\mathrm{CPO}$, cleft palate only; $\mathrm{C}$, the healthy control group.

miR-654-3p, miR-6515-5p, lncRNA RP11-731F5.2, lncRNA XIST, IncRNA RP11-591C20.9, RARA and SMPD1, as well as others. Through the analysis of the regulatory networks, we found that there may be a complex regulatory association between ncRNAs and mRNAs in the CL/P and CPO groups. Some of these key nodes and regulatory pairs need to be further validated in future studies.

Functional prediction of $n c R N A s$. It has been reported that lncRNAs can regulate the expression of target genes on multiple levels; however, the specific underlying mechanism remain unclear (33-36). In this study, the biological functions of IncRNAs were predicted by colocalization and co-expression. GO enrichment analysis was performed for the colocalization of target genes of DE IncRNAs between the CPO and control groups (Fig. 7A), the co-expression of target genes of DE lncRNAs between the CL/P and control groups (Fig. 7B), the co-expression target genes of DE lncRNAs between the $\mathrm{CPO}$ and control groups (Fig. 7C), and the target genes of DE miRNAs between the CPO and control groups (Fig. 7D). However, no significant enrichment $(\mathrm{P}>0.05)$ was found in the GO analysis of the target genes of DE miRNAs (Table SI) and in the colocalization of target genes of DE lncRNAs (Table SII) between the CL/P and control groups; thus, GO enrichment analysis figures could not be obtained. The genes with significant enrichment for each GO term were statistically analyzed and are displayed as a histogram. The results of the analysis indicated that the functions of dysregulated ncRNAs in the CPO group compared to the control group were mainly associated with 'immune response', 'immune system process', 'antigen processing and presentation', 'MHC protein complex' (Fig. 7A), 'intracellular', 'protein binding', 'cellular metabolic process', 'metabolic process' (Fig. 7C), 'cytoplasm', 'protein transport', 'secretion by cell', 'cellular localization' (Fig. 7D), as well as others. Additionally, the functions of dysregulated ncRNAs in the CL/P group compared to the control group were mainly associated with 'intracellular part', 'cytoplasmic part', 'protein binding', 'cellular metabolic process', 'metabolic process', 'gene expression', 'cellular localization', 'organic substance metabolic process', 'regulation of metabolic process', and others (Fig. 7B).

In this study, KEGG pathway analysis was performed for the target genes of DE lncRNAs and the target genes of DE miRNAs between the CL/P and control group and between 

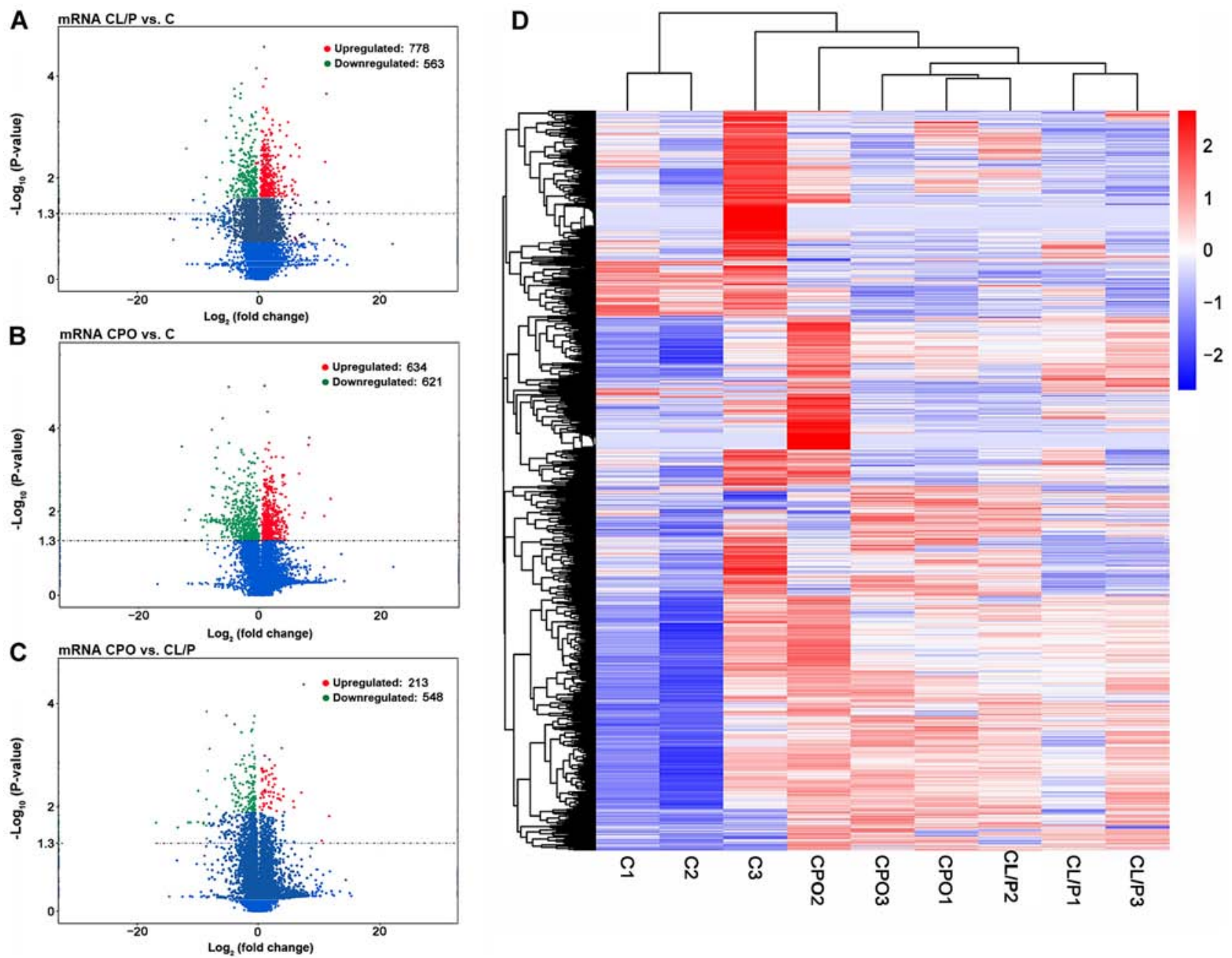

Figure 3. Transcriptome profile of RNA-Seq data of the mRNAs for the 3 groups. (A) Volcano plots of DE mRNAs between the CL/P and control groups, (B) between the CPO and control groups, and (C) between the CL/P and CPO groups. Red and green dots represent upregulated and downregulated mRNAs, respectively. (D) Hierarchical clustering of the expression profiles of DE mRNAs in the control, CL/P and CPO groups. CL/P, cleft lip with or without cleft palate; CPO, cleft palate only; $\mathrm{C}$, the healthy control group.

the CPO and control group. The KEGG pathway enrichment was analyzed with an enrichment criterion of P-value $<0.05$. However, only the analysis of the colocalization of target genes of DE lncRNAs between the CPO group and control group presented significant enrichment (Table IX). The rich factor refers to the ratio of the number of DE genes in the pathway to the total number of genes in the annotated gene list of the pathway. When the rich factor is greater and the $\mathrm{P}$-value is closer to zero, the enrichment is more significant. The results revealed that the most significantly involved pathways in the CPO group were 'antigen processing and presentation', 'phagosome', 'allograft rejection', 'type I diabetes mellitus', 'intestinal immune network for IgA production', 'viral myocarditis', 'rheumatoid arthritis' and 'ABC transporters'.

\section{Discussion}

This study first identified transcriptome profiles, including mRNAs, IncRNAs and miRNAs, from the peripheral blood of patients with $\mathrm{CL} / \mathrm{P}$ and $\mathrm{CPO}$ using a high-throughput sequencing method. Subsequently, the potential functions of the DE ncRNAs were predicted using bioinformatics tools and databases, and based on the theory of ceRNAs, we constructed IncRNA-miRNA-mRNA regulatory networks to identify the possible regulatory mechanisms between ncRNAs and mRNAs in NSOC.

Peripheral blood is the only tissue in the body that has contact with all organs. It carries RNA, DNA, vesicles and other biological substances that can aid clinicians in the diagnosis and identification of a variety of diseases, and its use is also more convenient and less invasive than other tissues $(37,38)$. Although the pathogenesis of NSOC is related to a number of factors during embryonic development, it has been found that a number of these differences persist after birth (39). In this study, the peripheral blood of patients with CL/P and CPO was used for high-throughput sequencing in an attempt to find DE ncRNAs compared to healthy individuals and to explore the possible regulatory mechanisms, although there were some limitations and differences in using peripheral blood instead of tissue samples due to ethical limitations. This study lays the foundation for future research, and the key DE ncRNAs and regulatory associations in NSOC need to be investigated in future studies. 
Table III. Detailed information of the top 10 differentially expressed microRNAs in the cleft lip with or without cleft palate group compared to the control group.

\begin{tabular}{lcc}
\hline microRNA & Log fold change & P-value \\
\hline hsa-let-7b-5p & 3.119 & $1.42 \times 10^{-6}$ \\
hsa-miR-3200-5p & 2.913 & $8.58 \times 10^{-7}$ \\
hsa-miR-150-3p & 2.387 & $2.60 \times 10^{-12}$ \\
hsa-miR-3200-3p & 2.378 & $3.40 \times 10^{-5}$ \\
hsa-miR-511-5p & 2.362 & $2.07 \times 10^{-4}$ \\
hsa-miR-376c-3p & -3.365 & $7.32 \times 10^{-8}$ \\
hsa-miR-655-3p & -2.964 & $2.91 \times 10^{-6}$ \\
hsa-miR-483-3p & -2.889 & $1.08 \times 10^{-7}$ \\
hsa-miR-4467 & -2.836 & $2.51 \times 10^{-6}$ \\
hsa-miR-561-5p & -2.680 & $1.29 \times 10^{-5}$ \\
\hline
\end{tabular}

Table IV. Detailed information of the top 10 differentially expressed microRNAs in the cleft palate only group compared to the control group.

\begin{tabular}{lcc}
\hline microRNA & Log fold change & P-value \\
\hline hsa-let-7b-5p & 4.458 & $2.29 \times 10^{-10}$ \\
hsa-miR-3200-5p & 3.831 & $2.89 \times 10^{-10}$ \\
hsa-miR-3200-3p & 3.590 & $8.30 \times 10^{-9}$ \\
hsa-let-7i-3p & 3.425 & $1.95 \times 10^{-11}$ \\
novel_859 & 3.365 & $1.32 \times 10^{-7}$ \\
hsa-miR-379-5p & -4.144 & $5.06 \times 10^{-10}$ \\
hsa-miR-136-3p & -4.040 & $1.34 \times 10^{-10}$ \\
hsa-miR-382-3p & -3.987 & $1.37 \times 10^{-13}$ \\
hsa-miR-381-3p & -3.901 & $1.27 \times 10^{-9}$ \\
hsa-miR-483-3p & -3.754 & $6.71 \times 10^{-11}$ \\
\hline
\end{tabular}

Due to ethical limitations, there have been no studies to date (at least to the best of our knowledge) on the expression profiles of ncRNAs of lip or palate tissues of humans. In a previous study, a research group used microarrays to screen the expression profiles of NSOC, and the results revealed that there were 305, 221 and 132 DE miRNAs in the plasma of patients with CLO, CL/P and CPO, respectively $(40,41)$. In those studies, miRNA-483-3p was significantly downregulated in both the CL/P and CPO groups, exhibiting a similar trend as that observed in this study. Although there have been no reports to date on miR-483-3p in NSOC (to the best of our knowledge), matrix metalloproteinase (MMP)9, the target gene of miR-483-3p, was found to be related to palate development. MMPs comprise a group of likely candidate proteins involved in the etiology of CL/P due to their role in modeling craniofacial tissues. The temporospatial expression of MMPs 2, 3, 7, 9 and 13 has been observed during murine palatal fusion (42-44). MMP9 is known for its ability to degrade type IV collagen, a main component of the extracellular matrix (ECM), and to facilitate cell migration (45). In a recent study, researchers analyzed miRNA expression in cultured palate fibroblasts
Table V. Detailed information of the top 10 differentially expressed long noncoding RNAs in the cleft lip with or without cleft palate group compared to the control group.

\begin{tabular}{|c|c|c|c|}
\hline Transcript_id & $\begin{array}{l}\text { Gene } \\
\text { name }\end{array}$ & $\begin{array}{l}\text { Log fold } \\
\text { change }\end{array}$ & P-value \\
\hline ENST00000483854.5 & RP11-706O15.1 & 6.580 & 0.0006 \\
\hline ENST00000441255.6 & RP11-81H14.2 & 4.675 & 0.0298 \\
\hline ENST00000460164.1 & RP11-731F5.2 & 4.334 & 0.0352 \\
\hline ENST00000614046.1 & RP11-147L13.15 & 1.930 & 0.0004 \\
\hline LNC_000209 & - & 1.802 & 0.0070 \\
\hline ENST00000625589.2 & RP11-70C1.3 & $-\operatorname{Inf}$ & 0.0046 \\
\hline ENST00000534671.1 & SBF2-AS1 & -5.301 & 0.0132 \\
\hline LNC_000567 & - & -3.852 & 0.0049 \\
\hline ENST00000525636.5 & SBF2-AS1 & -3.360 & 0.0079 \\
\hline LNC_002128 & - & -2.752 & 0.0183 \\
\hline
\end{tabular}

-Inf, negative infinity.

Table VI. Detailed information of the top 10 differentially expressed long noncoding RNAs in the cleft palate only group compared to the control group.

\begin{tabular}{lccr}
\hline Transcript_id & $\begin{array}{c}\text { Gene } \\
\text { name }\end{array}$ & $\begin{array}{c}\text { Log fold } \\
\text { change }\end{array}$ & P-value \\
\hline ENST00000460164.1 & RP11-731F5.2 & 6.545 & 0.0251 \\
LNC_000913 & - & 6.409 & 0.0450 \\
ENST00000494270.6 & RPARP-AS1 & 3.877 & 0.0076 \\
ENST00000429829.5 & XIST & 3.214 & 0.0189 \\
LNC_000039 & - & 2.172 & 0.0169 \\
ENST00000425031.1 & LINC00278 & -Inf & 0.0209 \\
LNC_000630 & - & - Inf & $<0.0001$ \\
LNC_002654 & - & -8.699 & 0.0164 \\
LNC_002129 & - & -5.345 & 0.0035 \\
ENST00000430245.5 & GAS5 & -4.969 & 0.0042 \\
\hline
\end{tabular}

-Inf, negative infinity.

from patients with CL/P and CPO using high-throughput sequencing methods. The results revealed that there were $9 \mathrm{DE}$ miRNAs in the CPO group, including miR-93-5p, miR-18a-5p, miR-92a-3p, miR-29c-5p, miR-549a, miR-3182, miR-181a-5p, miR-451a and miR-92b-5p. Patients with CL/P had only one DE miRNA (miR-505-3p) compared to the control group (46). In this study, the expression of miRNA-92b-5p was found to be also significantly downregulated in the CPO group compared to the control group. At present, miR-92b-5p has been studied mainly in tumors, cardiovascular and cerebrovascular diseases (47-50); however, its mechanism of action has rarely been reported. Based on the analysis of the results of this study with a target gene prediction software program, it should be noted that the expression of IncRNA XIST, which is the target gene of miR-92b-5p, was significantly upregulated in the CPO group. Although the regulatory association 
Table VII. Detailed information of the top 10 differentially expressed mRNAs in the cleft lip with or without cleft palate group compared to the control group.

\begin{tabular}{llrr}
\hline Transcript_id & \multicolumn{1}{c}{$\begin{array}{c}\text { Gene } \\
\text { name }\end{array}$} & $\begin{array}{c}\text { Log fold } \\
\text { change }\end{array}$ & P-value \\
\hline ENST00000611510 & RNASEH2B & 11.530 & 0.0294 \\
ENST00000299204 & BAG5 & 11.256 & 0.0002 \\
ENST00000397528 & SLC7A7 & 11.036 & 0.0048 \\
ENST00000451881 & CUL4A & 9.472 & 0.0300 \\
ENST00000451801 & ZNF683 & 6.928 & 0.0336 \\
ENST00000416778 & CPNE1 & -11.918 & 0.0026 \\
ENST00000608083 & ACOT7 & -10.826 & 0.0270 \\
ENST00000345506 & STAT5A & -9.041 & 0.0208 \\
ENST00000399338 & ITSN1 & -8.950 & 0.0298 \\
ENST00000605337 & MRPL37 & -8.721 & 0.0008 \\
\hline
\end{tabular}

Table VIII. Detailed information of the top 10 differentially expressed mRNAs in the cleft palate only group compared to the control group.

\begin{tabular}{llrr}
\hline Transcript_id & $\begin{array}{c}\text { Gene } \\
\text { name }\end{array}$ & $\begin{array}{c}\text { Log fold } \\
\text { change }\end{array}$ & P-value \\
\hline ENST00000299204 & BAG5 & 11.721 & 0.0050 \\
ENST00000376619 & HDAC6 & 10.693 & 0.0128 \\
ENST00000439744 & HVCN1 & 8.190 & 0.0002 \\
ENST00000473745 & IRF5 & 8.080 & 0.0002 \\
ENST00000554215 & DCAF5 & 7.436 & 0.0108 \\
ENST00000302779 & PXK & -12.868 & 0.0003 \\
ENST00000564243 & EIF3C & -12.316 & 0.0161 \\
ENST00000428575 & XRCC6 & -12.309 & 0.0491 \\
ENST00000636026 & ZEB2 & -11.635 & 0.0090 \\
ENST00000519501 & FAXDC2 & -9.719 & 0.0159 \\
\hline
\end{tabular}

between lncRNA XIST and miR-92b-5p in NSOC has not yet been reported, at leas to the best of our knowledge, other studies have found that lncRNA XIST and miR-92b directly interact with and repress each other, and lncRNA XIST inhibits hepatocellular carcinoma cell proliferation and metastasis by targeting miR-92b (50). Since the same regulatory relationship can be involved in multiple pathways, we speculated whether the IncRNA XIST-miR-92b relationship functions in the pathogenesis of CPO. These genes need to be verified in future experiments. Additionally, we found that some of the DE ncRNAs (or their target genes) and some DE mRNAs, including miR-96-5p, miR-92b-5p, miR-200b-3p, CD44, ZEB2, runt related transcription factor 3 (RUNX3), SMAD2, lncRNA RP11-731F5.2, and others, were reported in previous studies on NSOC or in related experiments. The present study found that T-Box 1 (TBX1) regulated the proliferation of dental progenitor cells and craniofacial development through miR-96-5p and paired-like homeodomain transcription factor 2 (PITX2). Additionally, TBX1, as a candidate gene of NSOC, can maintain the normal growth and development of palatal shelves, mediated through the regulation of genes involved in muscle cell differentiation, nervous system development and biomineral tissue development (51). The present study hypothesized that the dysregulated expression of miR-96-5p may affect the development of palatal shelves by inhibiting the expression of its target gene. CD44 is an integral cell membrane glycoprotein that acts as a receptor for ECM molecules involved in cell-cell interactions and cell adhesion and migration, and plays a postulated role in the proliferation of mesenchymal cells (52). Park et al (53) also found that CD44, as a candidate gene for $\mathrm{CL} / \mathrm{P}$, exhibited significant evidence of linkage in the presence of disequilibrium in 58 case-parent trios from Maryland and it was also found to be expressed in the developing palate and lip. RUNX3/PEBP $2 \alpha \mathrm{C}$ has multiple functions and was first found to be associated with the pathogenesis and progression of human gastric cancer as a tumor suppressor. The expression of RUNX3 was also observed in mouse embryonic palate and palatal shelf epithelium, which has been reported to express TGF $\beta 3$, bone morphogenetic protein (BMP)2 and BMP4 (54). SMAD2, a crucial regulator during palatal fusion, has been reported in a number of studies on NSOC. SMAD2 is necessary for the induction of Snail in TGF $\beta$-mediated epithelial-mesenchymal transition (EMT) at the cellular level $(55,56)$, and the cleft palates of TGF 33 -knockout mice were shown to be 'rescued' by the overexpression of SMAD2 in medial edge epithelia (MEE) cells (57). Another study found that miR-200b was expressed in the development of palatal shelves in mice and played a crucial role in regulating SMAD2 in apoptosis during palatogenesis by acting as a direct negative regulator (58). In addition, Shin et al (59) found that the ZEB family may be involved in cell migration during palate development and that excessive levels of miR-200b can lead to non-fused medial edge epithelium seam (MES) through the inhibition of ZEB1 and ZEB2 during palatogenesis. In this study, the expression of miR-200b-3p was upregulated, that of SMAD2 was downregulated and that of ZEB2 was also downregulated in the CPO group. It was thus hypothesized that miR-200b-3p may interact with ZEB2 or SMAD2, which play significant roles in the development of the palate.

Based on previous studies on the expression profiles of miRNAs in NSOC, this study adopted the high-throughput sequencing of peripheral blood of patients with CL/P and CPO to identify DE ncRNAs and mRNAs. The results of this study differ from those of previous studies, although there are still a few overlaps. It was hypothesized that this was due to the following reasons: First, we used different sample sources, resulting in different expression profiles. Second, the high-throughput sequencing method is more full-scale than the microarray method, which is more responsive to low-abundance transcripts. Finally, the false-positive results from high-throughput sequencing technology and analysis methods vary with each individual due to the different choices of software, databases and thresholds (60-62).

According to the fold changes, P-values and previous studies on NSOC, we selected 6 genes for further validation by RT-qPCR method with another 27 cases (10 controls, $10 \mathrm{CL} / \mathrm{P}$ and $7 \mathrm{CPO}$ ). The results revealed that the expression levels of 5 of these genes differed significantly and were 

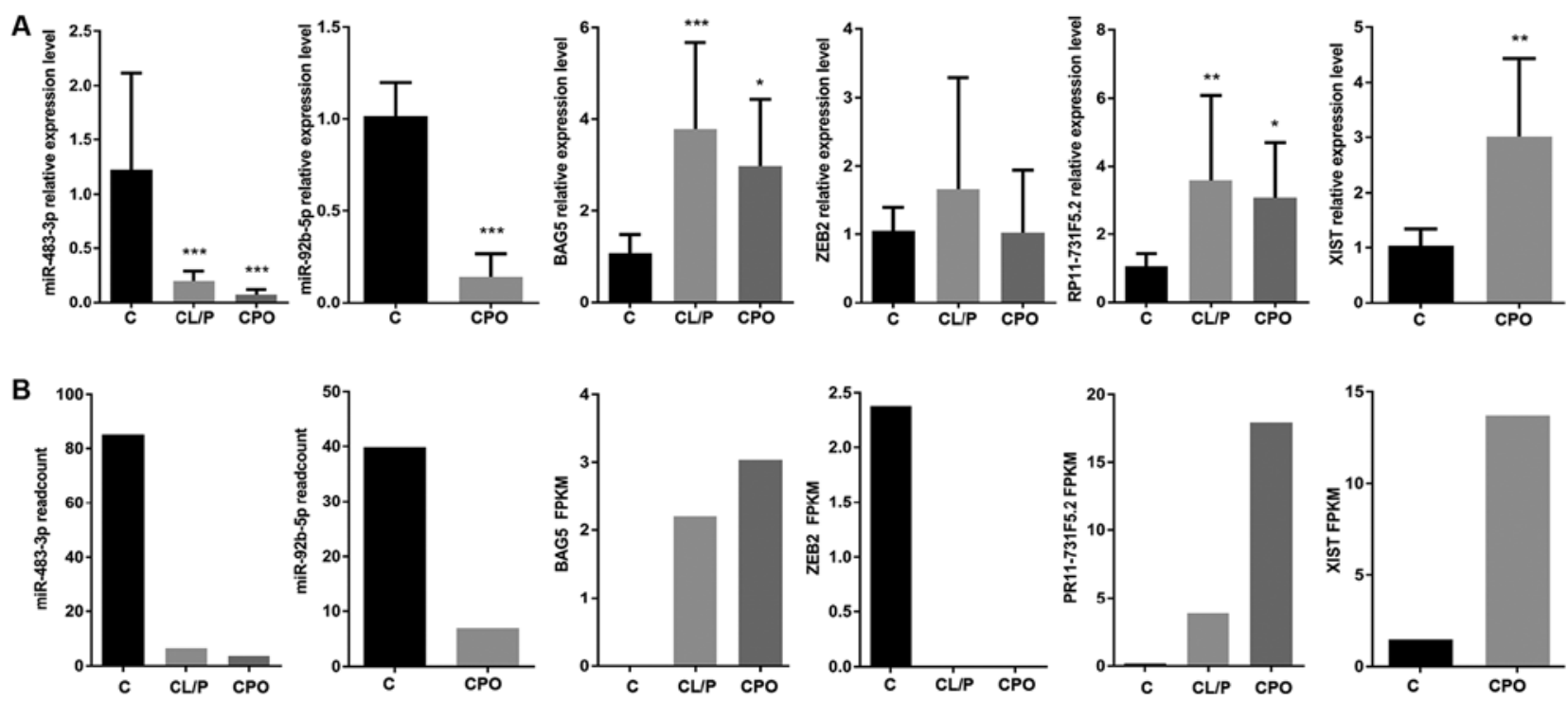

Figure 4. (A) The RT-qPCR validations of DE ncRNAs and mRNAs in the control, CL/P and CPO groups. The vertical axis shows the relative expression level of each RNA measured by RT-qPCR, ${ }^{*} \mathrm{P}<0.05,{ }^{* *} \mathrm{P}<0.01$ and ${ }^{* * * *} \mathrm{P}<0.001$, compared to the control. (B) The sequencing results for corresponding mRNAs, miRNAs and lncRNAs with qPCR verification. CL/P, cleft lip with or without cleft palate; CPO, cleft palate only; $\mathrm{C}$, healthy control group; RT-qPCR, quantitative real-time polymerase chain reaction; FPKM, fragments per kilobase million.

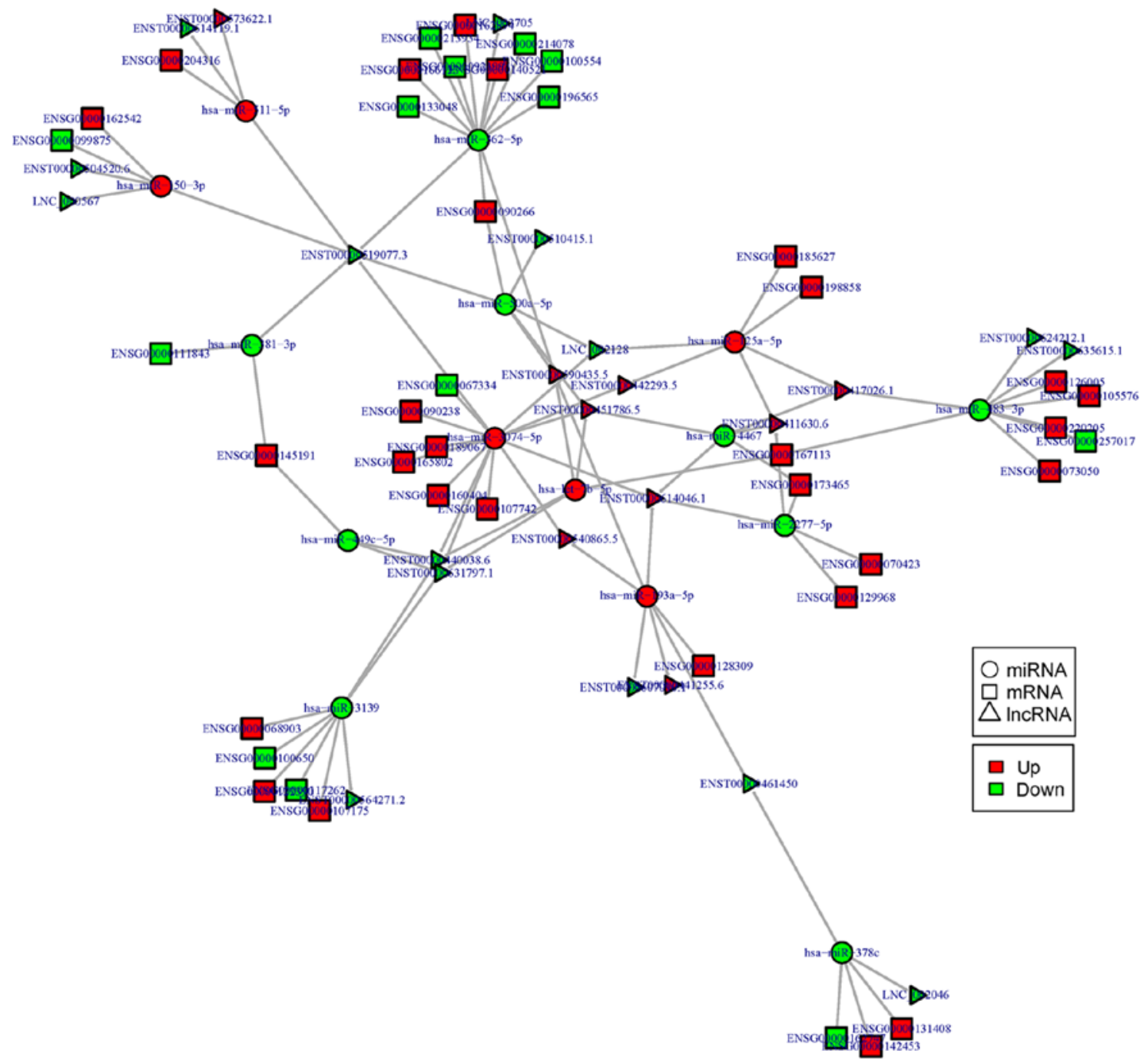

Figure 5. IncRNA-miRNA-mRNA regulatory network analysis of ncRNAs in the CL/P group. Different shapes represent different RNA types, and the different colors represent different regulatory relationships. Node size is proportional to the degree to which more edges are connected to the node, with large node size therefore indicating a greater the degree of relationship. IncRNA, long non-coding RNA; miRNA, microRNA; CL/P, cleft lip with or without cleft palate. 


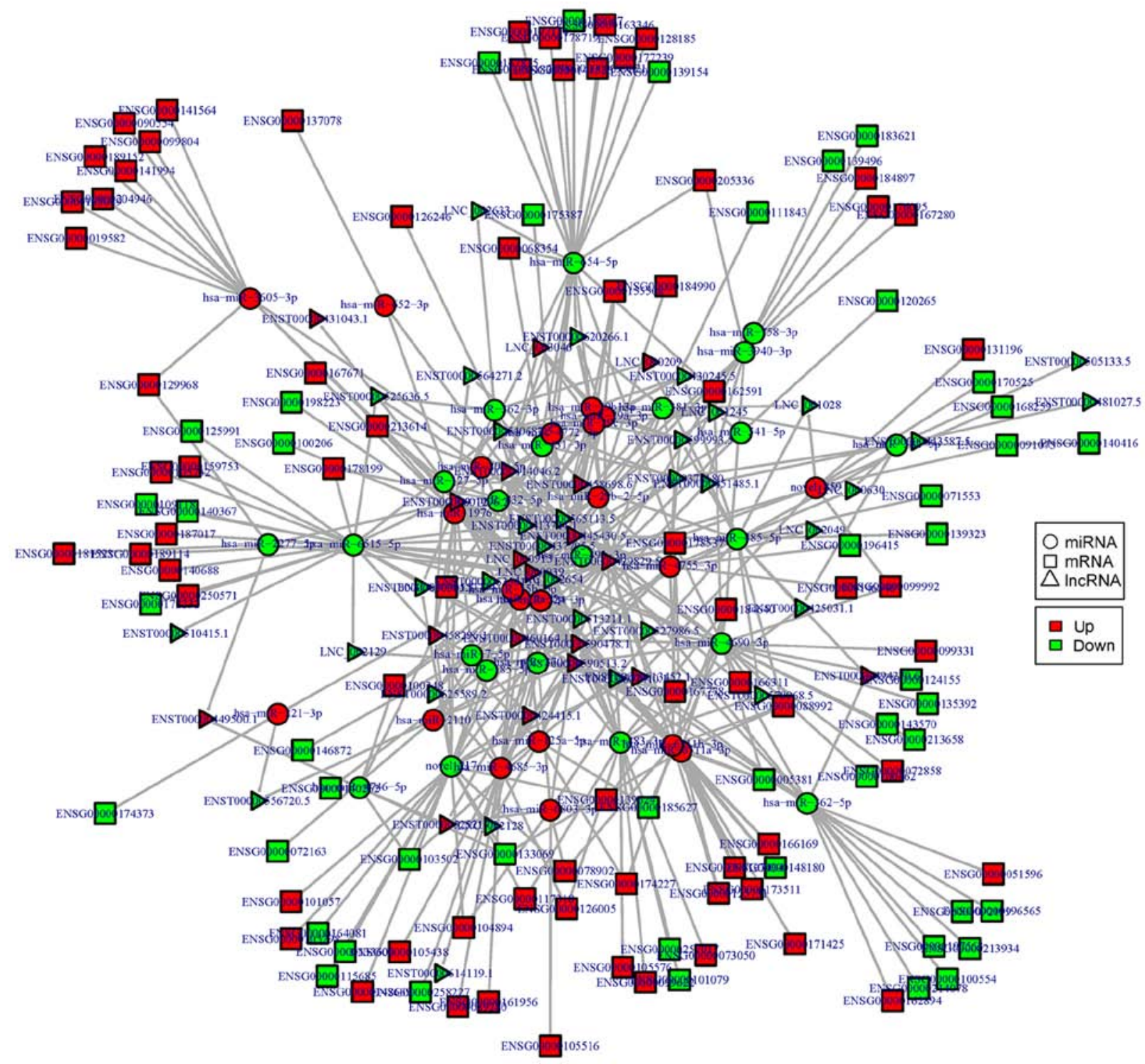

Figure 6. IncRNA-miRNA-mRNA regulatory network analysis of ncRNAs in the CPO group. Different shapes represent different RNA types, and the different colors represent different regulatory relationships. Node size is proportional to the degree to which more edges are connected to the node, with large node size therefore indicating a greater the degree of relationship. lncRNA, long non-coding RNA; miRNA, microRNA; CPO, cleft palate only.

consistent with the trends displayed by the sequencing results, apart from ZEB2, illustrating that the sequencing results are reliable and can provide a reference for further study. In addition, it was hypothesized that the following reasons account for the different trends in ZEB2 expression in the RT-qPCR and sequencing results. First, the samples were derived from human peripheral blood, which carries a large amount of genetic information and may lead to large differences between individuals. Second, the number of cases for sequencing was small, and false-positive results obtained with next-generation sequencing technology cannot be ruled out. Therefore, further in vitro or in vivo experiments are warranted in order to validate the present findings.

The primary and secondary palates have different embryonic sources; thus, orofacial clefts are generally divided into $\mathrm{CL} / \mathrm{P}$ and $\mathrm{CPO}$. The results of this study demonstrated that there were 19, 18 and 761 DE miRNAs, IncRNAs and mRNAs, respectively, in the $\mathrm{CPO}$ group compared to the $\mathrm{CL} / \mathrm{P}$ group, reflecting the separate etiologies of these 2 groups. Among the identified miRNAs, Warner et al (63) previously found that miR-199a-3p was expressed in the murine medial nasal and maxillary processes, which may be involved in the development of the lip and palate. Additionally, RUNX1 and ZEB1, which are the target genes of miR-199a-3p, have been reported to be associated with cleft lip and palate. Runx 1 previously has been shown to be important for the fusion of the primary and secondary palates (64). ZEB1 has been shown to regulate EMT in a number of systems, including the fusion of the secondary palate; however, there are no studies available to date examining its potential role in the fusion of the facial prominences (65). Thus, the potential role of miR-199a-3p in CL/P or CPO warrants further investigation in vivo or in vitro in the future. It worth noting that there were 5 overlapping genes between the $\mathrm{CL} / \mathrm{P}$ and control groups and between the $\mathrm{CPO}$ and control groups, including miR-7b-5p, miR-3200-5p, miR-3200-3p, miR-483-3p and lncRNA RP11-731F5.2. Yu et al (8) conducted a NSCLP GWAS using case-control samples from China and replicated 14 novel and 12 reported NSCLP risk loci in a total 
A
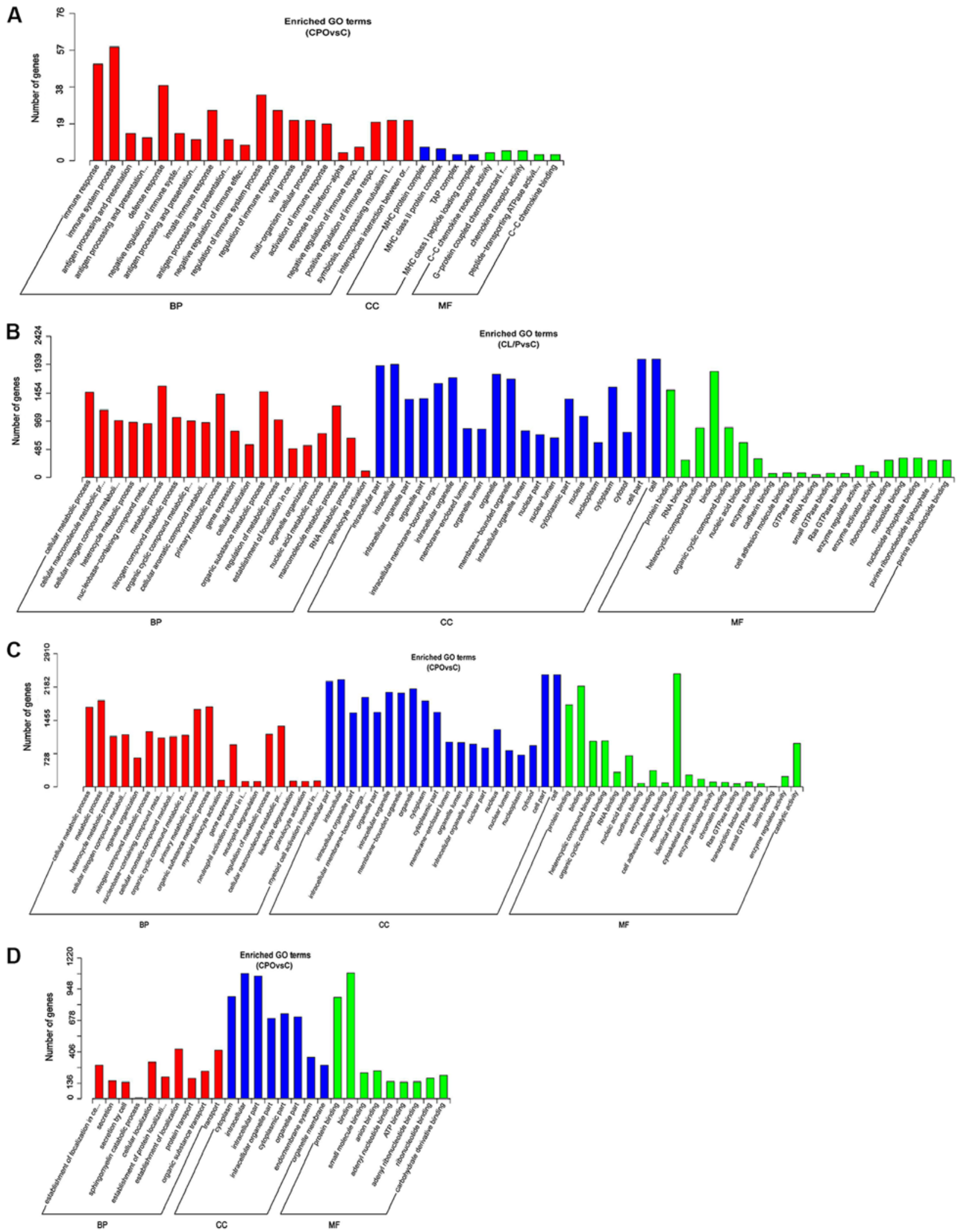

Figure 7. (A) GO analysis of colocalization target genes of DE lncRNAs between the CPO and control groups; (B) GO analysis of the co-expression target genes of DE lncRNAs between the CL/P and control groups; (C) GO analysis of the co-expression target genes of DE lncRNAs between the CPO and control groups; and (D) GO analysis of the target genes of DE miRNAs between the CPO and control groups. The horizontal axis represents the GO terms, with biological process (BP), cell component (CC), and molecular function (MF) from left to right, and the vertical axis is the number of genes annotated to each term. IncRNA, long non-coding RNA; CL/P, cleft lip with or without cleft palate; $\mathrm{CPO}$, cleft palate only; $\mathrm{C}$, the healthy control group.

of 23,463 samples from sub-phenotypes of NSOFC. They found that 1 novel and 2 reported NSCLP risk loci exhibited significant associations with CPO (8). This result indicated that the 2 sub-groups appeared to share distinct etiologies, although the overlapping risk factors between the 2 groups cannot be ruled out. Therefore, the overlapping genes need to 
Table IX. Kyoto Encyclopedia of Genes and Genomes pathway analysis of colocalization target genes of differentially expressed long noncoding RNAs in the cleft palate only group compared to the control group.

\begin{tabular}{lccc}
\hline Pathway_term & Rich_factor & P-value & Gene_number \\
\hline Antigen processing and presentation & 0.101 & 0.0019 & 8 \\
Phagosome & 0.065 & 0.0032 & 10 \\
Allograft rejection & 0.128 & 0.0097 & 5 \\
Graft-versus-host disease & 0.116 & 0.0107 & 5 \\
Type I diabetes mellitus & 0.111 & 0.0107 & 5 \\
Intestinal immune network for IgA production & 0.102 & 0.0127 & 5 \\
Autoimmune thyroid disease & 0.093 & 0.0160 & 5 \\
Asthma & 0.125 & 0.0160 & 4 \\
Staphylococcus aureus infection & 0.088 & 0.0160 & 5 \\
Viral myocarditis & 0.083 & 0.0171 & 5 \\
Rheumatoid arthritis & 0.066 & 0.0171 & 6 \\
ABC transporters & 0.091 & 0.0310 & 4 \\
Leishmaniasis & 0.068 & 0.0310 & 5 \\
Herpes simplex infection & 0.043 & 0.0310 & 8 \\
\hline
\end{tabular}

be further analyzed, for example, miR-483-3p and lncRNA RP11-731F5.2, mentioned above.

Salmena proposed the ceRNA hypothesis, which suggests that RNA molecules with the same miRNA response elements (MREs), including mRNAs, lncRNAs, pseudogenes and circRNAs, can regulate the expression abundance and translation activity of target genes by competitively binding to the same miRNA (66). The ceRNA causes protein-coding genes and ncRNAs to form complex and elaborate regulatory networks within the transcriptome and has been confirmed in diverse biological processes, including the differentiation of embryonic stem cells, the growth of the midbrain, cancer metastasis and others $(67,68)$. In this study, based on the ceRNA hypothesis, IncRNA-miRNA-mRNA regulatory networks were constructed to explore the potential regulatory associations between ncRNAs and mRNAs in NSOC. Crucial ncRNAs and mRNAs with more edges may play important roles in the pathology of CPO and CL/P. miR-483-3p and IncRNA XIST, mentioned above, were also the key nodes of the network in the CPO group. Additionally, lncRNA RP11-731F5.2 and its target gene, RARA, were found to have more edges in the network of the CPO group. Previous studies have reported that retinoic acid receptor $\alpha$ (RARA) may be a candidate gene for NSOC, and retinoic acid also may bind to RARA to inhibit apoptosis, resulting in the failure of palatal shelves fusion, whereas others have proposed that retinoic acid disrupts elevation or retards the growth of palatal shelves (69-71).

In this study, GO and KEGG pathway enrichment analysis was used to evaluate the biological functions and pathways of the target genes of DE ncRNAs. Through GO enrichment analysis of the target genes, we found that the function of $\mathrm{DE}$ ncRNAs in the $\mathrm{CPO}$ and $\mathrm{CL} / \mathrm{P}$ groups was mainly related to cellular behaviors, including 'intracellular part', 'cell adhesion molecule binding', 'cytoplasmic part', 'cellular metabolic process', 'cellular localization' and others. These cellular functions are coordinated by numerous genes encoding various growth factors, signaling mediators, transcriptional factors, cytokines, and extracellular matrix proteins (72). As is widely known, various cellular and molecular events are related to palatogenesis, including apoptosis, EMT, cell proliferation and cell migration, and any perturbation of these programs may cause NSOC. Therefore, it is crucial to explore genes and molecules that regulate the above-mentioned biofunctions in order to understand cellular behavior during palatogenesis.

The KEGG enrichment analysis revealed that the most significantly involved pathways in the $\mathrm{CPO}$ group were 'antigen processing and presentation', 'phagosome', 'allograft rejection', 'type I diabetes mellitus', 'intestinal immune network for IgA production', 'viral myocarditis', 'rheumatoid arthritis', and 'ABC transporters'. The etiology of NSOC is complex and related to multiple factors, suggesting that its development may involve other physiological systems and biological processes. According to a long-term follow-up survey, patients with cleft lip and palate have a higher risk of cancer, cardiovascular disease, central nervous system disease, and even suicide later in life compared to healthy individuals, although the underlying mechanisms have not been studied in depth (73). It was hypothesized that patients with cleft lip and palate may carry variations that do not cause other abnormalities at birth, but may increase the susceptibility to other diseases later on in life. Based on the data from the analysis of this study, an association between the risk of NSOC and the target genes enriched in other functions and signaling pathways cannot be ruled out.

Next-generation RNA sequencing was performed to identify the transcriptome profiles, including mRNAs, lncRNAs and miRNAs, in patients with CL/P and CPO. Subsequently, GO and KEGG enrichment analysis was performed to predict the functions of the DE ncRNAs; however, there are still some limitations. First, high-throughput sequencing technology itself has errors, and false-positive results can also be obtained from the analysis methods and prediction software. In addition, the exact mechanisms of action of the 
ncRNAs should be verified in vitro or in vivo. Second, when data was analyzed, WGCNA analysis was tentatively used to determine the correlation of IncRNA with mRNA. WGCNA analysis is a method based on a correlation coefficient, which is suitable for multi-sample analysis. According to previous studies, it is recommended that the sample size should be no less than 25 , and the more samples there are, the more reliable the results will be $(74,75)$. In this study, 9 samples were used for RNA-Seq ( 3 controls, $3 \mathrm{CL} / \mathrm{P}$ and $3 \mathrm{CPO}$ ). Given the above-mentioned considerations, WGCNA analysis was not performed in this study. Instead, a threshold of colocalization of $100 \mathrm{~kb}$ upstream and downstream of the lncRNAs was used. Pearson correlation coefficients with absolute values $>0.95$ were used to concurrently analyze the correlation between IncRNAs and mRNAs. However, if samples are sufficient in subsequent studies, the WGCNA analysis will be used to obtain more thorough results. Finally, the use of peripheral blood as a sample for sequencing is different than using tissue samples due to ethical limitations. Thus, this study provides only initial results regarding the pathogenesis of NSOC in epigenetics and lays the foundation for future verification using tissues. Additional studies may lead to the identification of novel diagnostic markers and therapeutic targets for NSOC.

\section{Acknowledgements}

Not applicable.

\section{Funding}

The present study was supported by the Innovation Research Project of Harbin Medical University (grant no. YJSCX2017-51HYD), and the National Key Research and Development Program of China (grant no. 2016YFC1000504).

\section{Availability of data and materials}

The datasets used and/or analyzed during the current study are available from the corresponding author on reasonable request.

\section{Authors' contributions}

$\mathrm{XJ}, \mathrm{SF}$ and WS were responsible for the design of the study. XW, QZ and YL diagnosed the patients with NSOC and collected the patients' peripheral blood. HS and WZ analyzed the data. YG performed the experiments and drafted the manuscript.

\section{Ethics approval and consent to participate}

The present study was approved by the Ethics Committee of Harbin Medical University, Heilongjiang, China and informed consent was obtained from the patients' families before sampling.

\section{Patient consent for publication}

Not applicable.

\section{Competing interests}

The authors declare that they have no competing interests.

\section{References}

1. Mossey PA, Little J, Munger RG, Dixon MJ and Shaw WC: Cleft lip and palate. Lancet 374: 1773-1785, 2009.

2. Leslie EJ and Marizita ML: Genetics of cleft lip and cleft palate. Am J Med Genet C Semin Med Genet 163C: 246-258, 2013.

3. Ladd-Acosta $\mathrm{C}$ and Beaty TH: Integrating RNA expression identifies candidate gene for orofacial clefts. J Dent Res 97: 31-32, 2018.

4. Chiquet BT, Hashmi SS, Henry R, Burt A, Mulliken JB, Stal S, Bray M, Blanton SH and Hecht JT: Genomic screening identifies novel linkages and provides further evidence for a role of MYH9 in nonsyndromic cleft lip and palate. Eur J Hum Genet 17: 195-204, 2009.

5. Chiquet BT, Yuan Q, Swindell EC, Maili L, Plant R, Dyke J, Boyer R, Teichgraeber JF, Greives MR, Mulliken JB, et al: Knockdown of Crispld2 in zebrafish identifies a novel network for nonsyndromic cleft lip with or without cleft palate candidate genes. Eur J Hum Genet 26: 1441-1450, 2018

6. Ludwig KU, Mangold E, Herms S, Nowak S, Reutter H, Paul A, Becker J, Herberz R, AlChawa T, Nasser E, et al: Genome-wide meta-analyses of nonsyndromic cleft lip with or without cleft palate identify six new risk loci. Nat Genet 44: 968-971, 2012.

7. Beaty TH, Murray JC, Marazita ML, Munger RG, Ruczinski I, Hetmanski JB, Liang KY, Wu T, Murray, T, Fallin, et al: A genome-wide association study of cleft lip with and without cleft palate identifies risk variants near MAFB and ABCA4. Nat Genet 42: 525-529, 2010.

8. Yu Y,Zuo X, He M, Gao J, Fu Y, Qin C, Meng L, Wang W, Song Y, Cheng Y, et al: Genome-wide analyses of non-syndromic cleft lip with palate identify 14 novel loci and genetic heterogeneity. Nat Commun 8: 14364, 2017.

9. Schoen C, Aschrafi A, Thonissen M, Poelmans G, Von den Hoff JW and Carels CEL: MicroRNAs in palatogenesis and cleft palate. Front Physiol 8: 165, 2017.

10. Moreno-Moya JM, Vilella F and Simón C: MicroRNA: Key gene expression regulators. Fertil Steril 101: 1516-1523, 2014.

11. Zhou J, Xiong Q, Chen H, Yang C and Fan Y: Identification of the spinal expression profile of Non-coding RNAs involved in neuropathic pain following spared nerve injury by sequence analysis. Front Mol Neurosci 10: 91, 2017.

12. Huang YK and Yu JC: Circulating microRNAs and long non-coding RNAs in gastric cancer diagnosis: An update and review. World J Gastroenterol 21: 9863-9886, 2015.

13. Zhao B, Lu M, Wang D, Li H and He X: Genome-wide identification of long noncoding RNAs in human intervertebral disc degeneration by RNA sequencing. Biomed Res Int 2016: $3684875,2016$.

14. Mukhopadhyay P, Brock G, Pihur V, Webb C, Pisano MM and Greene RM: Developmental microRNA expression profiling of murine embryonic orofacial tissue. Birth Defects Res A Clin Mol Teratol 88: 511-534, 2010.

15. Ding HL, Hooper JE, Batzel P, Eames BF, Postlethwait JH, Artinger KB and Clouthier DE: MicroRNA profiling during craniofacial development: Potential roles for Mir23b and Mir133b. Front Physiol 7: 281, 2016.

16. Dey BK, Mueller AC and Dutta A: Long non-coding RNAs as emerging regulators of differentiation, development, and disease. Transcription 5: e944014, 2014.

17. Ozturk F, Li Y, Zhu X, Guda C and Narwshad A: Systematic analysis of palatal transcriptome to identify cleft palate genes within TGF 33 -knockout mice alleles: RNA-Seq analysis of TGFß3 Mice. BMC Genomics 14: 113, 2013.

18. Gao L, Yin J and Wu W: Long non-coding RNA H19-mediated mouse cleft palate induced by 2,3,7,8-tetrachlorodibenzop-dioxin. Exp Ther Med 11: 2355-2360, 2016.

19. Gao L, Liu Y, Wen Y and Wu W: LncRNA H19-mediated mouse cleft palate induced by all-trans retinoic acid. Hum Exp Toxicol 36: 395-401, 2017.

20. Pertea M, Kim D, Pertea GM, Leek JT and Salzberg SL: Transcript-level expression analysis of RNA-seq experiments with HISAT, StringTie and Ballgown. Nat Protoc 11: 1650-1667, 2016. 
21. Pertea M, Pertea GM, Antonescu CM, Chang TC, Mendell JT and Salzberg SL: StringTie enables improved reconstruction of a transcriptome from RNA-seq reads. Nat Biotechnol 33: 290-295, 2015.

22. Sun L, Luo H, Bu D, Zhao G, Yu K, Zhang C, Liu Y, Chen R and Zhao Y: Utilizing sequence intrinsic composition to classify protein-coding and long non-coding transcripts. Nucleic Acids Res 41: e166, 2013.

23. Kong L, Zhang Y, Ye ZQ, Liu XQ, Zhao SQ, Wei L and Gao G: CPC: Assess the protein-coding potential of transcripts using sequence features and support vector machine. Nucleic Acids Res 35 (Web Server Issue): W345-W349, 2007.

24. Mistry J, Bateman A and Finn RD: Predicting active site residue annotations in the Pfam database. BMC Bioinformatics 8: 298, 2007.

25. Lin MF, Jungreis I and Kellis M: PhyloCSF: A comparative genomics method to distinguish protein coding and non-coding regions. Bioinformatics 27: i275-i282, 2011.

26. Langmead B, Trapnell C, Pop M and Salzberg SL: Ultrafast and memory-efficient alignment of short DNA sequences to the human genome. Genome Biol 10: R25, 2009.

27. Wen M, Shen Y, Shi S and Tang T: miREvo: An integrative microRNA evolutionary analysis platform for next-generation sequencing experiments. BMC Bioinformatics 13: 140, 2012.

28. Friedländer MR, Mackowiak SD, Li N, Chen W and Rajewsky N: miRDeep2 accurately identifies known and hundreds of novel microRNA genes in seven animal clades. Nucleic Acids Res 40 37-52, 2012.

29. Love MI, Huber W and Anders S: Moderated estimation of fold change and dispersion for RNA-seq data with DESeq2. Genome Biol 15: 550, 2014

30. Livak KJ and Schmittgen TD: Analysis of relative gene expression data using real-time quantitative PCR and the 2(-Delta Delta C(T)) method. Methods 25: 402-408, 2001.

31. Young MD, Wakefield MJ, Smyth GK and Oshlack A: Gene ontology analysis for RNA-seq: Accounting for selection bias. Genome Biol 11: R14, 2010.

32. Mao X, Cai T, Olyarchuk JG and Wei L: Automated genome annotation and pathway identification using the KEGG Orthology (KO) as a controlled vocabulary. Bioinformatics 21 3787-3793, 2005

33. Ponting CP, OliPver PL and Reik W: Evolution and functions of long noncoding RNAs. Cell 136: 629-641, 2009.

34. Nagano $T$ and Fraser P: No-nonsense functions for long noncoding RNAs. Cell 145: 178-181, 2011.

35. Wilusz JE, Sunwoo H and Spector DL: Long noncoding RNAs: Functional surprises from the RNA world. Genes Dev 23: 1494-1504, 2009.

36. Kopp F and Mendell JT: Functional classification and experimental dissection of long noncoding RNAs. Cell 172: 393-407, 2018.

37. Huan T, Joehanes R, Schurmann C, Schramm K, Pilling LC, Peters MJ, Mägi R, DeMeo D, O'Connor GT, Ferrucci L, et al: A whole-blood transcriptome meta-analysis identifies gene expression signatures of cigarette smoking. Hum Mol Genet 25 4611-4623, 2016

38. Hardy JJ, Mooney SR, Pearson AN, McGuire D, Correa DJ, Simon RP and Meller R: Assessing the accuracy of blood RNA profiles to identify patients with post-concussion syndrome: A pilot study in a military patient population. PLoS One 12 : e0183113, 2017.

39. Zhang J, Zhou S, Zhang Q, Feng S, Chen Y, Zheng H, Wang X Zhao W, Zhang T, Zhou Y, et al: Proteomic analysis of RBP4/vitamin A in children with cleft lip and/or palate. J Dent Res 93: 547-552, 2014

40. Li J, Zou J, Li Q, Chen L, Gao Y, Yan H, Zhou B and Li J: Assessment of differentially expressed plasma microRNAs in nonsyndromic cleft palate and nonsyndromic cleft lip with cleft palate. Oncotarget 7: 86266-86279, 2016.

41. Zou J, Li J, Li J, Ji C, Li Q and Guo X: Expression profile of plasma microRNAs in nonsyndromic cleft lip and their clinical significance as biomarkers. Biomed Pharmacother 82: 459-466, 2016.

42. Iamaroon A, Wallon UM, Overall CM and Diewert VM: Expression of 72-kDa gelatinase (matrix metalloproteinase-2) in the developing mouse craniofacial complex. Arch Oral Biol 41: $1109-1119,1996$

43. Morris-Wiman J, Du Y and Brinkley L: Occurrence and temporal variation in matrix metalloproteinases and their inhibitors during murine secondary palatal morphogenesis. J Craniofac Genet Dev Biol 19: 201-212, 1999.
44. Morris-Wiman J, Burch $\mathrm{H}$ and Basco E: Temporospatial distribution of matrix metalloproteinase and tissue inhibitors of matrix metalloproteinases during murine secondary palate morphogenesi. Anat Embryol (Berl) 202: 129-141, 2000.

45. Letra A, da Silva RA, Menezes R, de Souza AP, de Almeida AL, Sogayar MC and Granjeiro JM: Studies with MMP9 gene promoter polymorphism and nonsyndromic cleft lip and palate. Am J Med Genet A 143A: 89-91, 2007.

46. Schoen C, Glennon JC, Abghari S, Bloemen M, Aschrafi A, Carels CEL and Von den Hoff JW: Differential microRNA expression in cultured palatal fibroblasts from infants with cleft palate and controls. Eur J Orthod 40: 90-96, 2018.

47. Wu T, Chen Y, Du Y, Tao J, Zhou Z and Yang Z: Serum exosomal MiR-92b-5p as a potential biomarker for acute heart failure caused by dilated cardiomyopathy. Cell Physiol Biochem 46: 1939-1950, 2018

48. Wu T, Chen Y, Du Y, Tao J, Li W, Zhou Z and Yang Z: Circulating exosomal miR-92b-5p is a promising diagnostic biomarker of heart failure with reduced ejection fraction patients hospitalized for acute heart failure. J Thorac Dis 10: 6211-6220, 2018.

49. Zhao C, Zhao F, Feng H, Xu S and Qin G: MicroRNA-92b inhibits epithelial-mesenchymal transition-induced migration and invasion by targeting Smad3 in nasopharyngeal cancer. Oncotarget 8: 91603-91613, 2017.

50. Zhuang LK, Yang YT, Ma X, Han B, Wang ZS, Zhao QY, Wu LQ and Qu ZQ: MicroRNA-92b promotes hepatocellular carcinoma progression by targeting Smad7 and is mediated by long non-coding RNA XIST. Cell Death Dis 7: e2203, 2016.

51. Gao S, Moreno M, Eliason S, Cao H, Li X, Yu W, Bidlack FB, Margolis HC, Baldini A and Amendt BA: TBX1 protein interactions and microRNA-96-5p regulation controls cell proliferation during craniofacial and dental development: Implications for 22q11.2 deletion syndrome. Hum Mol Genet 24: 2330-2348, 2015.

52. Baroni T, Bellucci C, Lilli C, Pezzetti F, Carinci F, Lumare E, Palmieri A, Stabellini G and Bodo M: Human cleft lip and palate fibroblasts and normal nicotine-treated fibroblasts show altered in vitro expressions of genes related to molecular signaling pathways and extracellular matrix metabolism. J Cell Physiol 222: 748-756, 2010.

53. Park JW, Cai J, McIntosh I, Jabs EW, Fallin MD, Ingersoll R, Hetmanski JB, Vekemans M, Attie-Bitach T, Lovett M, et al: High throughput SNP and expression analyses of candidate genes for non-syndromic oral clefts. J Med Genet 43: 598-608, 2006.

54. Yamamoto H, Ito K, Kawai M, Murakami Y, Bessho K and Ito Y: Runx3 expression during mouse tongue and palate development. Anat Rec A Discov Mol Cell Evol Biol 288: 695-699, 2006.

55. Nawshad A, Medici D and Liu CC: TGFbeta3 inhibits E-cadherin gene expression in palate medial-edge epithelial cells through a Smad2-Smad4-LEF1 transcription complex. J Cell Sci 120: 1646-1653, 2007.

56. Nawshad A, LaGamba D and Hay ED: Transforming growth factor beta (TGFbeta) signalling in palatal growth, apoptosis and epithelial mesenchymal transformation (EMT). Arch Oral Biol 49: 675-689, 2004.

57. Cui XM, Shiomi N, Chen J, Saito T, Yamamoto T, Ito Y, Bringas P, Chai Y and Shuler CF: Overexpression of Smad2 in Tgf-beta3-null mutant mice rescues cleft palate. Dev Biol 278: 193-202, 2005

58. Shin JO, Lee JM, Cho KW, Kwak S, Kwon HJ, Lee MJ, Cho SW, Kim KS and Jung HS: MiR-200b is involved in Tgf- $\beta$ signaling to regulate mammalian palate development. Histochem Cell Biol 137: 67-78, 2012.

59. Shin JO, Nakagawa E, Kim EJ, Cho KW, Lee JM, Cho SW and Jung HS: miR-200b regulates cell migration via Zeb family during mouse palate development. Histochem Cell Biol 137: 459-470, 2012

60. Mutz KO, Heilkenbrinker A, Lönne M, Walter JG and Stahl F: Transcriptome analysis using next-generation sequencing. Curr Opin Biotechnol 24: 22-30, 2013

61. Mantione KJ, Kream RM, Kuzelova H, Ptacek R, Raboch J, Samuel JM and Stefano GB: Comparing bioinformatic gene expression profiling methods: Microarray and RNA-Seq. Med Sci Monit Basic Res 20: 138-142, 2014.

62. Lowe R, Shirley N, Bleackley M, Dolan S and Shafee T: Transcriptomics technologies. PLoS Comput Biol 13: e1005457, 2017.

63. Warner DR, Mukhopadhyay P, Brock G, Webb CL, Michele Pisano $\mathrm{M}$ and Greene RM: MicroRNA expression profiling of the developing murine upper lip. Dev Growth Differ 56: 434-447, 2014. 
64. Charoenchaikorn K, Yokomizo T, Rice DP, Honjo T, Matsuzaki K, Shintaku Y, Imai Y, Wakamatsu A, Takahashi S, Ito Y, et al: Runx1 is involved in the fusion of the primary and the secondary palatal shelves. Dev Biol 326: 392-402, 2009.

65. Liu Y, El-Naggar S, Darling DS, Higashi Y and Dean DC: Zeb1 links epithelial-mesenchymal transition and cellular senescence. Development 135: 579-588, 2008.

66. Salmena L, Poliseno L, Tay Y, Kats L and Pandolfi PP: A ceRNA hypothesis: The Rosetta Stone of a hidden RNA language? Cell 146: 353-358, 2011

67. Lai K, Jia S, Yu S, Luo J and He Y: Genome-wide analysis of aberrantly expressed lncRNAs and miRNAs with associated co-expression and ceRNA networks in $\beta$-thalassemia and hereditary persistence of fetal hemoglobin. Oncotarget 5: 49931-49943, 2017.

68. Tay Y, Rinn J and Pandolfi PP: The multilayered complexity of ceRNA crosstalk and competition. Nature 505: 344-352, 2014.

69. Dai J, Yu H, Si J, Fang B and Shen SG: Irf6-related gene regulatory network involved in palate and lip development. J Craniofac Surg 26: 1600-1605, 2015.

70. Choi JW, Park HW, Kwon YJ and Park BY: Role of apoptosis in retinoic acid-induced cleft palate. J Craniofac Surg 22: 1567-1571, 2011.
71. Chenevix-Trench G, Jones K, Green AC, Duffy DL and Martin NG: Cleft lip with or without cleft palate: Associations with transforming growth factor alpha and retinoic acid receptor loci. Am J Hum Genet 51: 1377-1385, 1992.

72. Zhu X, Ozturk F, Pandey S, Guda CB and Nawshad A: Implications of TGF $\beta$ on transcriptome and cellular biofunctions of palatal mesenchyme. Front Physiol 3: 85, 2012.

73. Christensen K, Juel K, Herskind AM and Murray JC: Long term follow up study of survival associated with cleft lip and palate at birth. BMJ 328: 1405, 2004.

74. Langfelder P and Horvath S: WGCNA: An R package for weighted correlation network analysis. BMC Bioinformatics 9: 559, 2008.

75. De Oliveira PSN, Coutinho LL, Tizioto PC, Cesar ASM, de Oliveira GB, Diniz WJDS, De Lima AO, Reecy JM, Mourão GB, Zerlotini A and Regitano LCA: An integrative transcriptome analysis indicates regulatory mRNA-miRNA networks for residual feed intake in Nelore cattle. Sci Rep 8: 17072, 2018.

(i) $($ This work is licensed under a Creative Commons Attribution-NonCommercial-NoDerivatives 4.0 International (CC BY-NC-ND 4.0) License. 